\title{
Lung ultrasound for diagnosis and monitoring of ventilator-associated pneumonia
}

\author{
Bélaïd Bouhemad $^{1,2}$, Ophélie Dransart-Rayé ${ }^{1,2}$, Francesco Mojoli $^{3,4}$, Silvia Mongodi ${ }^{4}$ \\ ${ }^{1}$ Department of Anesthesiology and Intensive Care, C.H.U. Dijon, France; ${ }^{2}$ Dijon and Université Bourgogne Franche-Comté, LNC UMR866, \\ Dijon, France; ${ }^{3}$ Department of Clinical-Surgical, Diagnostic and Pediatric Sciences, Unit of Anaesthesia and Intensive Care, University of Pavia, \\ Pavia, Italy; ${ }^{4}$ Anesthesia and Intensive Care, Fondazione IRCCS Policlinico San Matteo, Pavia, Italy \\ Contributions: (I) Conception and design: All authors; (II) Administrative support: None; (III) Provision of study materials or patients: None; (IV) \\ Collection and assembly of data: None; (V) Data analysis and interpretation: None; (VI) Manuscript writing: All authors: All authors; (VII) Final \\ approval of manuscript: All authors. \\ Correspondence to: Prof. Bélaïd Bouhemad. Service d'Anesthésie Réanimation CHU Dijon and Université Bourgogne Franche-Comté, LNC \\ UMR866, F-21000 Dijon, France. Email: belaid_bouhemad@hotmail.com.
}

\begin{abstract}
Ventilator-associated pneumonia (VAP) is the most frequent nosocomial infection in intensive care units (ICU) and is associated with increased mortality, use of antimicrobials, longer mechanical ventilation, and higher healthcare costs. Lung ultrasonography (LUS) can be used at the bedside and gained widespread acceptance in ICU. Although the visualization of a single LUS sign cannot be considered specific for a diagnosis, clinically-driven LUS examination in particular setting and clinical conditions allow ruling in or out quickly and accurately several causes of acute respiratory failure. This article reviews LUS signs for VAP diagnosis and summarizes the studies testing LUS for VAP diagnosis and monitoring. Many VAP occurs in already injured regions, thus presence of lobar consolidation is not enough to affirm VAP. However, a linear/arborescent air-bronchogram confirms the diagnosis of VAP with a good specificity, a normal LUS rules out the diagnosis of VAP (in experimented hands). LUS, thanks to its bedside ready availability, has the potential to become a key tool in early VAP diagnosis. LUS could ideally represent the decision-making tool for antimicrobial therapy administration in the timeframe of the technical time required for bronchoalveolar lavage analysis. A systematic approach for diagnosis and monitoring of VAP with LUS is also proposed in this review. But specific data on LUS specificity and sensitivity for the diagnosis of VAP are still lacking and should be investigated.
\end{abstract}

Keywords: Diagnostic imaging; echography; ventilator associated pneumonia (VAP); lung monitoring; lung ultrasonography (LUS)

Submitted Oct 09, 2018. Accepted for publication Oct 16, 2018.

doi: $10.21037 /$ atm.2018.10.46

View this article at: http://dx.doi.org/10.21037/atm.2018.10.46

\section{Introduction}

Ventilator-associated pneumonia (VAP) is the most frequent nosocomial infection in intensive care units (ICUs) and is associated with increased mortality, use of antimicrobials, longer mechanical ventilation, and higher healthcare costs $(1,2)$. In developing countries, VAP occurs in $30 \%$ of patients under mechanical ventilation and is a leading cause of death, being associated to $16-94 \%$ mortality $(3,4)$.

In histopathological studies, VAP is characterized by an infiltration of neutrophils, fibrin exudates and cellular debris into the intra-alveolar spaces, particularly around terminal bronchioles, caused by the invasion of the bacterial pathogens (5). In clinical practice, VAP diagnosis relies usually on a microbiological sample of distal airways (6). However, reliability of these samples for differentiating lung parenchyma invasion from lower airway colonization is still debated. Moreover a sample requires 24 to 48 hours for preliminary/definitive results. 
Table 1 Clinical pulmonary infection score (CPIS)

\begin{tabular}{|c|c|}
\hline Parameter & CPS \\
\hline \multicolumn{2}{|l|}{ Temperature $\left({ }^{\circ} \mathrm{C}\right)$} \\
\hline$\geq 36.5$ and $\leq 38.5$ & 0 \\
\hline$\geq 38.5$ and $\leq 38.9$ & 1 \\
\hline$\geq 39$ and $\leq 36.0$ & 2 \\
\hline \multicolumn{2}{|l|}{ Blood leukocytes per $\mathrm{mm}^{3}$} \\
\hline$\geq 4,000$ or $\leq 11,000$ & 0 \\
\hline$<4,000$ or $>11,000$ & 1 \\
\hline \multicolumn{2}{|l|}{ Tracheal secretions } \\
\hline Few & 0 \\
\hline Moderate & 1 \\
\hline Large & 2 \\
\hline Purulent & +1 \\
\hline \multicolumn{2}{|l|}{ Oxygenation $\mathrm{PaO}_{2} / \mathrm{FiO}_{2}, \mathrm{mmHg}$} \\
\hline$>240$ or presence of ARDS & 0 \\
\hline$\leq 240$ and absence of ARDS & 2 \\
\hline \multicolumn{2}{|l|}{ Chest radiograph } \\
\hline No infiltrate & 0 \\
\hline Patchy or diffuse infiltrate & 1 \\
\hline Localized infiltrate & 2 \\
\hline \multicolumn{2}{|l|}{${ }^{*}$ EA quantitative culture } \\
\hline No or light quantity & 0 \\
\hline Moderate/heavy quantity & 1 \\
\hline $\begin{array}{l}\text { Same pathogenic bacteria of Gram } \\
\text { stain }\end{array}$ & 2 \\
\hline \multicolumn{2}{|c|}{$\begin{array}{l}\text { *, additional parameters to be added in for modified versions of } \\
\text { the score. ARDS, acute respiratory distress syndrome; } \mathrm{PaO}_{2} / \\
\mathrm{FiO}_{2} \text {, arterial oxygen partial pressure/fraction of inspired oxygen; } \\
\mathrm{CFU} \text {, colony forming unit; } \mathrm{EA} \text {, endotracheal aspirate. }\end{array}$} \\
\hline
\end{tabular}

To this day, there is no consensus in early VAP diagnosis; deciding whether to treat a patient is often subjective and debated within teams when a VAP is clinically suspected on the basis of clinical, biochemical and imaging parameters (7). In such patients, inappropriate or delayed antibiotics lead to higher mortality ( $64.5 \%$ vs. $29.2 \%$ ) (8). On the other hand, overexposure to antibiotic also results in increased morbi-mortality and is responsible for the emergence of bacterial resistance. Therefore, an accurate and early diagnosis of VAP is crucial to reach the optimal balance between mortality reduction and multi-drug resistance prevention $(8,9)$.

Among imaging techniques, chest radiography (CXR) is widely used despite its limited performance in VAP diagnosis $(10,11)$. Computed tomography (CT) remains the gold standard $(12,13)$. However, CT implies patient transportation to radiology department, radiation exposure and costs. Point-of-care ultrasound is a non-invasive nonirradiating technique performed at the bedside; its use has largely spread in the last years in critical care, making this technique widely available in ICU (14-16). Lung ultrasonography (LUS) helps in the differential diagnosis of acute respiratory failure when integrated in clinically driven protocols (17). Moreover, LUS features make it suitable for multiple repeated examinations, therefore providing a powerful monitoring tool.

This article reviews criteria for VAP diagnosis and summarizes the studies testing ultrasound application for VAP diagnosis and monitoring.

\section{Diagnosis of VAP}

VAP is suspected when a new radiographic infiltrate develops in a patient with fever/hypothermia, leukocytosis/ leukopenia, purulent tracheal secretions, and impaired oxygenation. A combination of clinical criteria [Clinical Pulmonary Infection Score (CPIS)] has been proposed for VAP diagnosis (Table 1) (7). However, many non-infectious processes can cause fever and pulmonary infiltrate in patients under mechanical ventilation, making the diagnosis of VAP difficult (18). Previous study shows CPIS poor diagnostic performance, with low specificity and sensibility, thus limiting its daily use (19). This is also true when the CPIS is enhanced by direct gram-stain examination of the tracheal aspirate and/or procalcitonin (20).

Quantitative bacterial cultures of specimen obtained from the lower airways using bronchoscopic and nonbronchoscopic techniques were proposed for helping in VAP diagnosis. A cut off value of $10^{4}$ colony-forming unit/ml has been set to identify positive samples; however, the presence of no or lower bacterial charge when antibiotics have been introduced or modified in the previous 48 hours also confirms pneumonia, in presence of all the suggestive clinical criteria.

Fiber-bronchoscopy is however not always easy to perform in hypoxemic patients and not promptly available in all ICUs; it could therefore be replaced by tracheal aspirate microbiological samples. Usefulness of tracheal 
aspirate microbiological samples has been investigated and they are considered in some countries a reliable alternative to distal samples. It is recommended that for most patients a clinical approach enhanced by non-invasive quantitative cultures of respiratory tract samples is sufficient to guide appropriate antibiotic choice $(21,22)$. However, this attitude is still debated.

Microbiological results are essential not only to confirm the diagnosis but also to target antibiotic therapy; they require 24 hours for preliminary results and 48 hours for definitive results and antibiogram. Therefore, microbiological cultures cannot guide the early clinical management of patients with a suspected VAP and deciding whether to start antibiotics remains a challenge

\section{Classical imaging in VAP}

Current guidelines recommend CXR and CT for VAP diagnosis $(12,13)$. However, in mechanically ventilated patients pulmonary infiltrates are frequent and may be associated to multiple etiologies, including non-infectious diseases such as de-recruitment and atelectasis. Imaging detection of VAP in critical care setting is therefore difficult, especially when a single morphological evaluation is performed, since providing limited information.

CXR has low diagnosis performances for pneumonia in the critically ill. Indeed, at ICU admission CXR is abnormal in $38 \%$ of mechanically ventilated patients (23). Furthermore, in a recent study evaluating CXR compared with CT in the emergency department, up to $30 \%$ false positive and false negative results were reported. Finally, portable CXR has limited resolution: pulmonary opacity specificity for the diagnosis of VAP has been reported to be as low as $27 \%$ to $35 \%(10,11)$.

$\mathrm{CT}$ provides a more precise interpretation of chest infiltrates in ICU. However, there is no specific CT sign of pneumonia described in mechanically ventilated (24) and CT is mostly used for its negative predictive value. In addition, CT is irradiating, less readily available and requires patients' transportation to radiology department, which may be harmful in the critically ill.

Thus, the interest in developing an alternative imaging technique for a reliable detection of pneumonia is high.

\section{Lung ultrasound signs}

\section{Lung examination}

Lung ultrasound examination is performed at the bedside in supine position directly by the intensivist (14). Ultrasound machines are nowadays available in most of the ICUs and both simple and advanced machines are suitable to perform lung ultrasound. For each hemithorax, 6 regions are examined: 3 areas are delimited from the front to the back by sternum and anterior and posterior axillary lines; each area is divided into a superior and an inferior region (Figure 1). The choice of the probe depends on patient morphology and suspected pathology. The examination can be started by anterior fields with a high-frequency linear probe, suitable for superficial examination and therefore for the visualization of the pleural line and derived artifacts; the operator can switch to a low-frequency probe (convex or phased-array) if real images are visualized (consolidations, effusions) or the pleural line is too deep to be assessed with a linear probe. A micro-convex probe, if available, is suitable for both types of examination.

\section{A-lines}

In healthy lung, air does not allow ultrasound penetration and only the pleural line is visualized. During tidal ventilation, the lungs expand and the visceral pleura slide against the parietal one, corresponding to a sparkling movement of the pleural line synchronous with inspiration (lung sliding). Beyond this pleural line, regularly spaced motionless horizontal lines (A-lines) are reverberation artifacts of the pleural line; they are generated when lung density is normal $(25,26)$. Normal lung aeration is defined by both lung sliding and A-lines.

\section{B-lines}

Abnormal interlobular septa or alveoli edema provide a gastissue interface with the pleural line that alters ultrasound reflection. The result is an ultrasound artifact known as B-line: a hyperechoic laser-shaped vertical artifact arising from the pleural line, moving synchronous with lung sliding, reaching the edge of the screen and erasing the A-lines. B-lines are generated when the lung density increases (25) and are considered significant when $\geq 3$ B-lines are visualized in a scan (B-pattern). $\geq 3$ well-spaced B-lines correspond to moderate lung loss of aeration whereas coalescent B-lines, resulting from partial filling of alveolar spaces, indicate severe loss of aeration (27). Those two patterns are classically observed in increased extra vascular lung water either due to increased hydrostatic pressure or altered capillary permeability (Figure 2). 


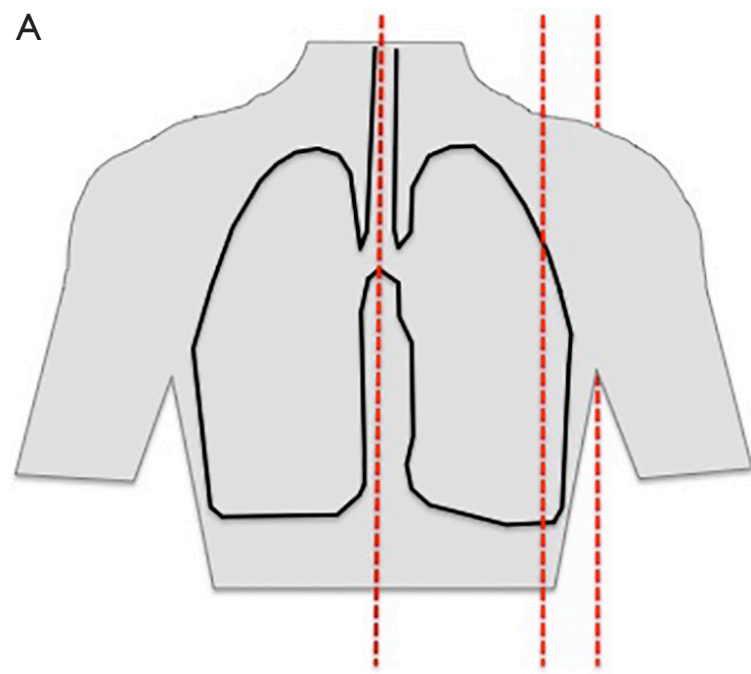

\begin{tabular}{|c|c|c|c|}
\hline AREA & SCORE & $\begin{array}{l}\text { Sub- } \\
\text { pleural }\end{array}$ & $\begin{array}{c}\text { Air- } \\
\text { Bronchogram }\end{array}$ \\
\hline L1 & & $\square$ & $\square$ \\
\hline L2 & & $\square$ & $\square$ \\
\hline L3 & & $\square$ & $\square$ \\
\hline L4 & & $\square$ & $\square$ \\
\hline L5 & & $\square$ & $\square$ \\
\hline L6 & & $\square$ & $\square$ \\
\hline R1 & & $\square$ & $\square$ \\
\hline R2 & & $\square$ & $\square$ \\
\hline R3 & & $\square$ & $\square$ \\
\hline R4 & & $\square$ & $\square$ \\
\hline R5 & & $\square$ & $\square$ \\
\hline R6 & & $\square$ & $\square$ \\
\hline TOTAL & & & \\
\hline
\end{tabular}

Figure 1 Lung ultrasound examination and report. (A) Each hemithorax is divided in six regions: 2 anterior, 2 lateral, 2 posterior, according to anatomical landmarks set by anterior and posterior axillary lines (red dotted lines). Each region is divided in half, superior, and inferior. To perform a comprehensive examination, all adjacent intercostal spaces must be explored in each region of interest, sliding the probe along the space. (B) Examples of simplified report form for rapid reporting and monitoring. For each explored region, the worst finding is reported in simple checkboxes according to the following rating: normal: 0; well-separated B-lines: 1; coalescent B-lines: 2; and consolidation: 3 . The cumulative lung ultrasound score corresponds to the sum of each examined region score (minimum score, normal lungs: 0 ; maximum score, both consolidated lungs: 36). ANT, anterior; INF, inferior; LAT, lateral; POST, posterior; SUP, superior.

\section{Lung consolidation}

Lung lobar/hemilobar consolidations are visualized when the loss of aeration is complete and the lung appears as a tissue-like structure. Lung consolidations have a welldelimited external margin, while the inner limit can be either irregular if aerated lung is in continuity or regular in case of complete lobe consolidation. Within consolidations, air-bronchograms are visualized as hyperechoic images. During inspiration, penetration of gas through the bronchial tree into consolidations reinforces their appearance, giving a dynamic airbronchogram. A dynamic air-bronchogram allows ruling out obstructive atelectasis (28). If the air-bronchogram is static or absent, it suggests non-patent airways: however, it is not specific for a diagnosis. Air-bronchogram morphology (punctiform or linear/arborescent) may provide additional information for further differential diagnosis of consolidations (Figures 3,4).

Smaller consolidations are visualized as echo-poor images juxtaposed to the pleura with irregular boundaries (shred sign), the so-called subpleural consolidations (Figures 3,5).

\section{Lung ultrasound for community acquired pneumonia (CAP) diagnosis}

In emergency departments, a recent study compared CXR to chest CT scan for the diagnosis of suspected CAP: CXR showed $30 \%$ false positive and false negative rate (31). On the opposite, LUS showed good results in CAP diagnosis. LUS visualization of a consolidation yielded a sensitivity of $93 \%$ and a specificity of $98 \%$ for the diagnosis of communityacquired pneumonia in the emergency department (32). The visualization of a lung consolidation with LUS was confirmed to be a valid alternative for the bedside diagnosis of CAP in adults in multiple successive studies (33-40). This was also affirmed by recent meta-analyses $(41,42)$.

Although the visualization of a single ultrasound sign cannot be considered specific for a diagnosis, clinicallydriven LUS examination in particular setting and clinical conditions allow ruling in or out quickly and accurately several causes of acute respiratory failure in $\operatorname{ED}(32,43,44)$. A recent single-blind, randomized controlled trial demonstrated that point-of-care ultrasound is superior to standard diagnostic tests alone for establishing a correct diagnosis of acute respiratory failure, also allowing a better 

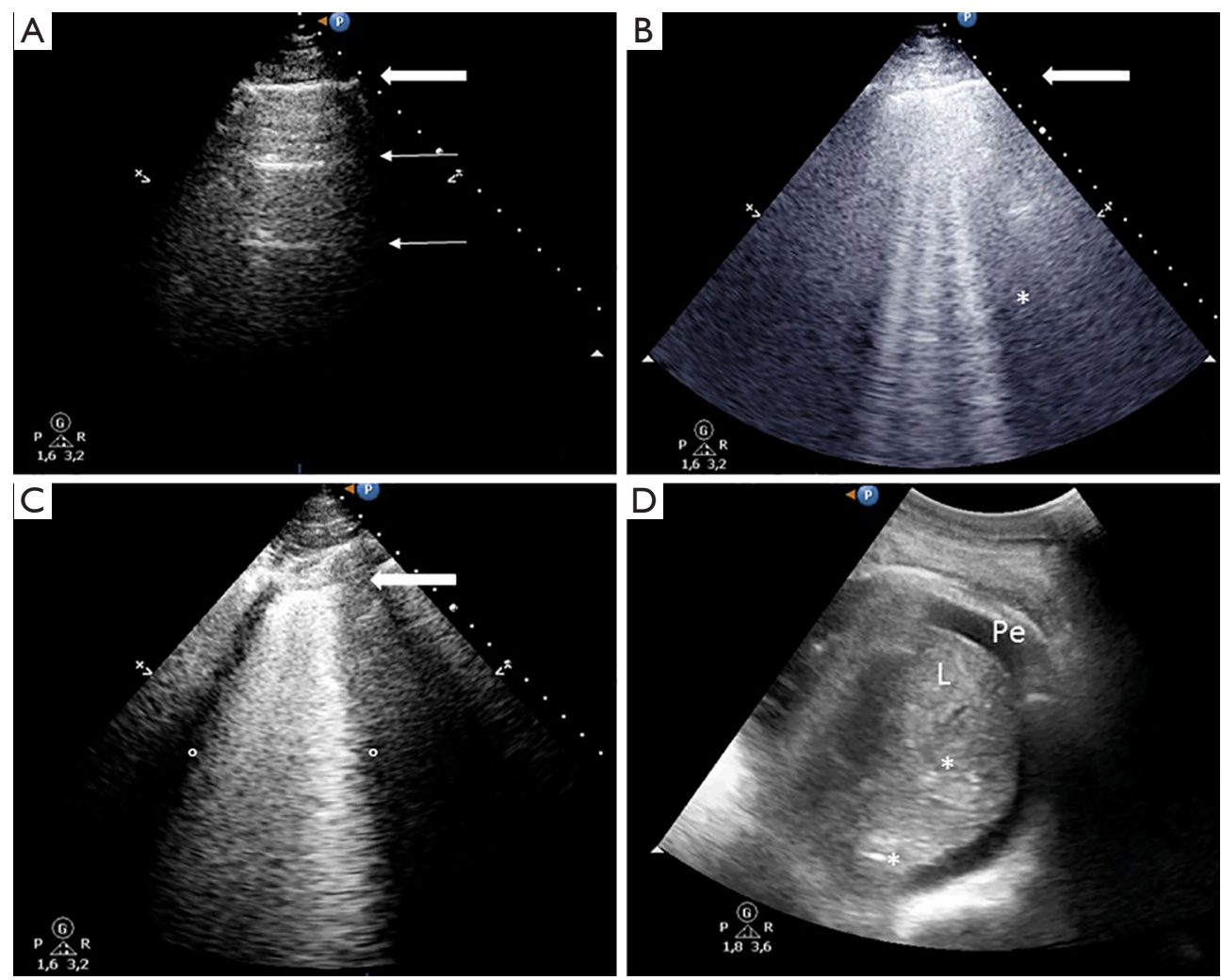

Figure 2 Lung ultrasound score patterns. (A) Normal pattern. The pleural line (white arrow) with multiple horizontal A-lines (thin arrows). (B) The pleural line is visible (white arrow) with separated B-lines $\left(^{*}\right)$ arising from the pleural line and spreading up to the edge of the screen. This pattern corresponds to moderate lung aeration loss resulting from interstitial syndrome. (C) The pleural line is visible (white arrow) with coalescent B-lines (between ${ }^{\circ}$ ) arising from the pleural line and spreading up to the edge of the screen. This pattern corresponds to severe lung aeration loss resulting from partial filling of alveolar spaces. (D) Transversal view of a consolidated lower lobe. Lung has a tissuelike echotexture (L). Hyperechoic images $\left(^{*}\right.$ ) can be seen within the consolidation corresponding to dynamic air bronchograms (air-filled bronchi). Pe, pleural effusion.

use of advanced diagnostic tests (17).

\section{Lung ultrasound for VAP diagnosis}

The diagnosis of VAP is trickier than the simple visualization of consolidations; in fact, patients under mechanical ventilation may present severe loss of aeration due to other etiologies, mainly in dependent regions.

LUS has all the features to be suitable for VAP detection, but only three original works focused so far on its application for VAP diagnosis. In a retrospective study by Zagli et al., they investigated the diagnostic value of chest infiltrates with ultrasound, considering both tissuelike pattern visualization and subpleural consolidation (45). Ultrasound was integrated in a complex score also including clinical parameters, PCT and quantitative microbiological analysis of endotracheal aspirate (Table 2). This score showed sensitivity $80.5 \%$ and specificity $85.2 \%$, but results were necessarily boosted by the integration in the score of the quantitative microbiological parameter. Moreover, problematic patients such as with other potential sources of infection, COPD decompensation or with ongoing antibiotics were excluded, thus limiting its applicability.

If considered alone, chest infiltrates showed acceptable specificity (84.6\%) but poor sensitivity (59.3\%), as expected. However, this corresponded to a first signal on the potential use of ultrasound visualization of chest infiltrates for VAP diagnosis.

A second study by Berlet et al focused on the monitoring of modifications of consolidations' pattern in patients 

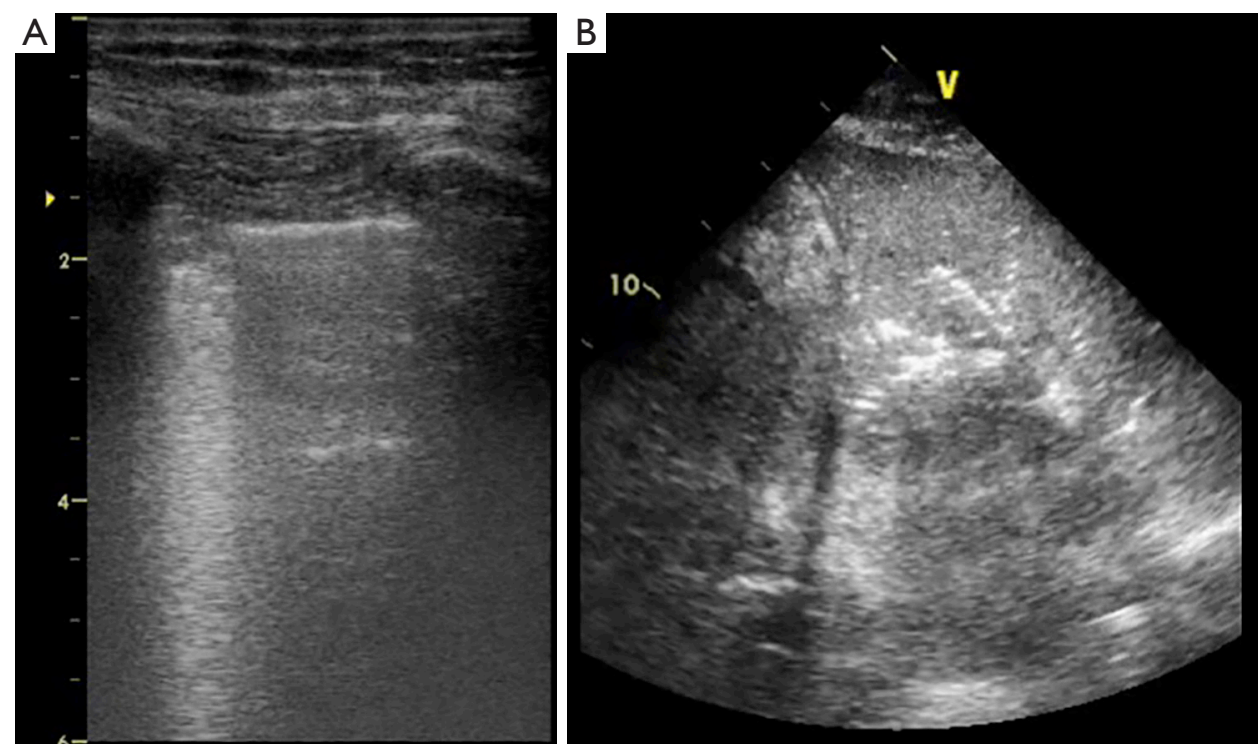

Figure 3 Lung ultrasound signs of VAP. (A) A subpleural consolidation is visualized on the left as an echopoor image juxtaposed to the pleura and generating hyperechoic artifacts (longitudinal scan - linear probe). (B) Within a consolidated lung, a dynamic arborescent airbronchogram is visualized as a hyperechoic image moving synchronous with tidal ventilation and shaping the bronchial tree.

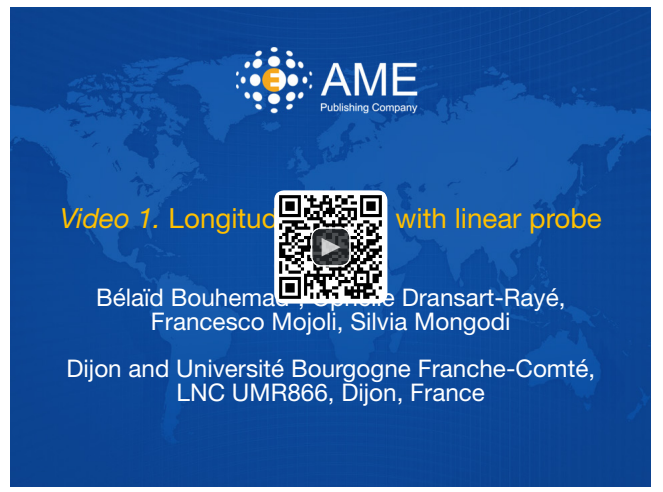

Figure 4 Longitudinal scan with linear probe (29): the bat sign is clearly visible; $0.5 \mathrm{~cm}$ below the rib line, the pleural line is visualized as a hyperechoic line, moving synchronous with tidal ventilation. A subpleural consolidation is visualized on the left as an echopoor image juxtaposed to the pleura and generating hyperechoic artifacts.

Available online: http://www.asvide.com/article/view/28249

considered at risk of VAP (46). While limited by the small sample size (only 12 patients affected by VAP) and by a simplified LUS examination (only posterior regions), an interest in the identification of dynamic air-bronchogram for VAP diagnosis was underlined.

A successive prospective multicenter study by Mongodi

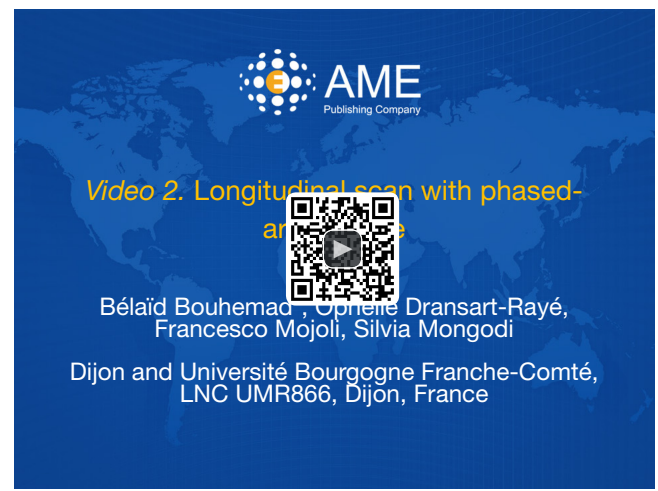

Figure 5 Longitudinal scan with phased-array probe (30): the diaphragm cupola is visualized separating abdominal parenchyma (on the left) and a consolidated lung (on the right). Within the lung, the hyperechoic image moving synchronous with tidal ventilation and shaping the bronchial tree corresponds to a dynamic arborescent air-bronchogram.

Available online: http://www.asvide.com/article/view/28250

et al. (47) enrolled 99 consecutive patients with suspected VAP on the basis of classical clinical and biochemical parameters; the performances of LUS, CPIS, direct gramstain examination and quantitative analysis of tracheal aspirate were compared to the gold standard, a distal airways microbiological sample. A complete 12-region 
Table 2 Chest echography and procalcitonin pulmonary infection score (CEPPIS)

\begin{tabular}{ll}
\hline Parameter & CEPPIS \\
\hline Temperature $\left({ }^{\circ} \mathrm{C}\right)$ & 0 \\
$\geq 36.0$ and $<38.4$ & 1 \\
$\geq 38.5$ and $<38.9$ & 2 \\
$\geq 39$ and $<36.0$ & \\
Procalcitonin $(\mathrm{ng} / \mathrm{mL})$ & 0 \\
$<0.5$ & 1 \\
$\geq 0.5$ and $<1$ & 2 \\
$\geq 1$ & \\
Tracheal secretions & 0 \\
Non purulent & 2 \\
Purulent & 2 \\
Oxygenation PaO $/$ FiO ${ }_{2}$, mmHg & \\
$>240$ or presence of ARDS & \\
$\leq 240$ and absence of ARDS & \\
Infiltrates on LUS & \\
Negative & \\
Positive & \\
$\leq 10^{4}$ CFU/mL & \\
\hline & \\
\hline &
\end{tabular}

*, additional parameters to be added in for modified versions of the score. ARDS, acute respiratory distress syndrome; $\mathrm{PaO}_{2} /$ $\mathrm{FiO}_{2}$, arterial oxygen partial pressure/fraction of inspired oxygen; CFU, colony forming unit; EA, endotracheal aspirate.

Table 3 Ventilator-associated pneumonia lung ultrasound score (VPLUS)

\begin{tabular}{lc}
\hline Parameter & VPLUS \\
\hline Purulent tracheal secretions & 1 \\
$\geq$ Areas with subpleural & 1 \\
consolidations & 2 \\
$\geq$ Area with dynamic linear/ & \\
arborescent air-bronchogram & 1 \\
EA positive quantitative/ & \\
qualitative culture* & \\
\hline
\end{tabular}

*, additional parameters to be added in for modified versions of the score. EA, endotracheal aspirate.
LUS examination was performed, focusing on the presence of consolidations (both tissue-like pattern and subpleural consolidations) and on the air-bronchogram features (present or absent, static or dynamic, punctiform or linear/ arborescent). The combination of clinical and biochemical parameter was confirmed to have poor performances for early VAP diagnosis: a CPIS $\geq 6$ carried $68 \%$ sensitivity and $50 \%$ specificity. Direct gram-stain examination and quantitative culture of tracheal aspirate showed 57\%/77\% and $83 \% / 71 \%$ of sensitivity/specificity, thus confirming a reliable but not perfect role of quantitative analysis of tracheal aspirate to confirm VAP diagnosis.

Concerning LUS signs, the presence of a tissue-like pattern alone had $93 \%$ sensitivity but $0 \%$ specificity, thus confirming that other etiologies than VAP may induce complete loss of aeration in mechanically ventilated patients. On the contrary, the visualization of one consolidated region with linear/arborescent dynamic airbronchogram showed greater specificity $(81 \%)$. Subpleural consolidations resulted to be a sensible sign, with limited specificity (at least one subpleural consolidation: sensitivity $81 \%$, specificity $41 \%$ ).

A new simple score, easy to compute at the bedside, was tested post-hoc, the VAP lung ultrasound score (VPLUS, Table 3): it's AUC was higher than the CPIS (0.743 vs. 0.574) and was improved by the association of direct gram-stain examination or quantitative analysis of endotracheal aspirate (0.832 and 0.874 , respectively). A VPLUS $\geq 2$ identified VAP with $71 \%$ sensitivity and $69 \%$ specificity.

In pathology, disseminated associated VAP injuries extend from the center to the periphery. Wherever those lesions reach the pleural line, they become identifiable by LUS. As VAP extends, normal pattern is replaced by focal areas of interstitial syndrome represented by well-spaced B lines, progressively becoming confluent; this is then replaced by small subpleural consolidations, disseminated to anterior and lateral chest wall. If not treated, those small consolidations merge to become larger lobar consolidation. The air-bronchogram might be visualized within large consolidations.

Being VAP an evolutive process reaching the chest wall, lung ultrasound examination seems to perfectly match VAP diagnosis features. However, despite the description of specific ultrasound patterns for VAP, to this day, a prospective application of LUS signs in early VAP diagnosis is lacking. Because many VAP occurs in already injured 
regions, we believe that the LUS examination should focus on lesions evolution rather than on appearance of new lesions.

To summarize LUS signs of VAP are given in Figure 3. In patients suspected of VAP, LUS can be a valuable help by visualizing lobar or subpleural consolidation quickly and reliably. A linear/arborescent air-bronchogram confirms the diagnosis of VAP. In experimented hands, a normal LUS rules out the diagnosis of VAP.

\section{Lung ultrasound for VAP monitoring}

Once diagnosed, LUS can also be used to monitor VAP recovery and antibiotic efficacy (48). Effective treatment should result in progressive disappearance of VAP signs whereas when inappropriately treated, subpleural consolidations are expected to keep appearing and to extend.

For this purpose, a LUS score can be used to quantify the global loss of aeration and monitor its modification in days. An aeration score is attributed to each of the 12 standard regions: 0 if A-lines or maximum 2 B-lines are visualized, score 1: $\geq 3 \mathrm{~B}$-lines (well-spaced or involving $\leq 50 \%$ of the pleura, depending on the approach), score 2: B-lines becoming coalescent or involving $>50 \%$ of the pleura, score 3: tissue-like pattern $(49,50)$. The global LUS score is computed as the sum of the regional scores; it showed good correlation with lung density as assessed by CT scan (25) and was so far applied to quantify and monitor aeration in weaning from mechanical ventilation (50), in acute respiratory distress syndrome (ARDS) on extra-corporeal membrane oxygenation (51) and to guide fluid resuscitation in ARDS patients in shock (52).

A regional re-aeration score can be computed in each area to monitor the effectiveness of a treatment aiming at increasing lung aeration, as antibiotics in $\operatorname{VAP}(46)$ or recruitment maneuvers (53): the aeration improvement will give a positive score of $+1,+3$ or +5 points if a switch of 1 , 2 or 3 steps in LUS score is observed. A worsening in lung aeration will give a negative score of $-1,-3$ or -5 points is a switch of 1,2 or 3 steps is remarked. The global re-aeration score is computed as the sum of each regional re-aeration score.

When computed after 7 days of antibiotics in VAP patients, global LUS re-aeration score showed a significant correlation with re-aeration as assessed by quantitative CT scan (rho $=0.85$ ). In particular, a re-aeration score equal or superior to 5 corresponded to a gain of lung volume of at least $400 \mathrm{~mL}$, corresponding to a successful antibiotic therapy, while a score inferior to -10 corresponded to a loss of aeration of at least $400 \mathrm{ml}$, thus indicating antibiotic failure. A systematic approach for diagnosis and monitoring of VAP with LUS is proposed in Figure 6.

Moreover, LUS score can be easily learnt with a 25 -exam training (54) and showed good repeatability both in terms of images acquisition (25) and images interpretation (49).

Non-resolution of infection with development of abscesses or septated effusions can also be detected (Figure 7). Abscesses with pleural contact or included inside a lung consolidation (55-58) appear as rounded hypo-echoic lesions with regular outer margins (Figure 3). If a cavity is present, additional B-lines are generated by the gas/tissue interface. Ultrasound-guided percutaneous drainage of lung abscesses has proven to be a safe and effective alternative to CT-guided drainage (55-58).

\section{Limitations}

Classical limitations in LUS application are the presence of subcutaneous emphysema or thoracic dressings, since preventing ultrasound penetration. For the specific application of VAP diagnosis and monitoring, although previous study suggested the presence of specific and sensible ultrasound signs, a prospective application is still lacking. Moreover, while the dynamic linear/arborescent air-bronchogram is a specific sign, it's poorly sensible and false negative exist; a second LUS examination after disobstructive fiber-bronchoscopy has been suggested to detect previously masked air-bronchogram, but still has to be confirmed in a wider population (59).

\section{Conclusions}

The routine use of LUS is now an attractive alternative to bedside CXR in diagnosing lung consolidation and pleural effusion, and its value is recognized in CAP diagnosis. On the other hand, accuracy of lung ultrasound for diagnosing of VAP is still under evaluation. In ICU patients with pre-existing injured lung areas, the presence of a lung consolidation is not enough to affirm VAP. LUS imaging can show lung infiltrate and sign of VAP, more reliably and quickly using LUS than chest X-ray, but it cannot attest the presence of lung bacteria in the lung and cannot replace microbiological sampling. However, it seems that some LUS signs are specific of lung infection and can accurately predict VAP when combined to simple clinical parameters. 


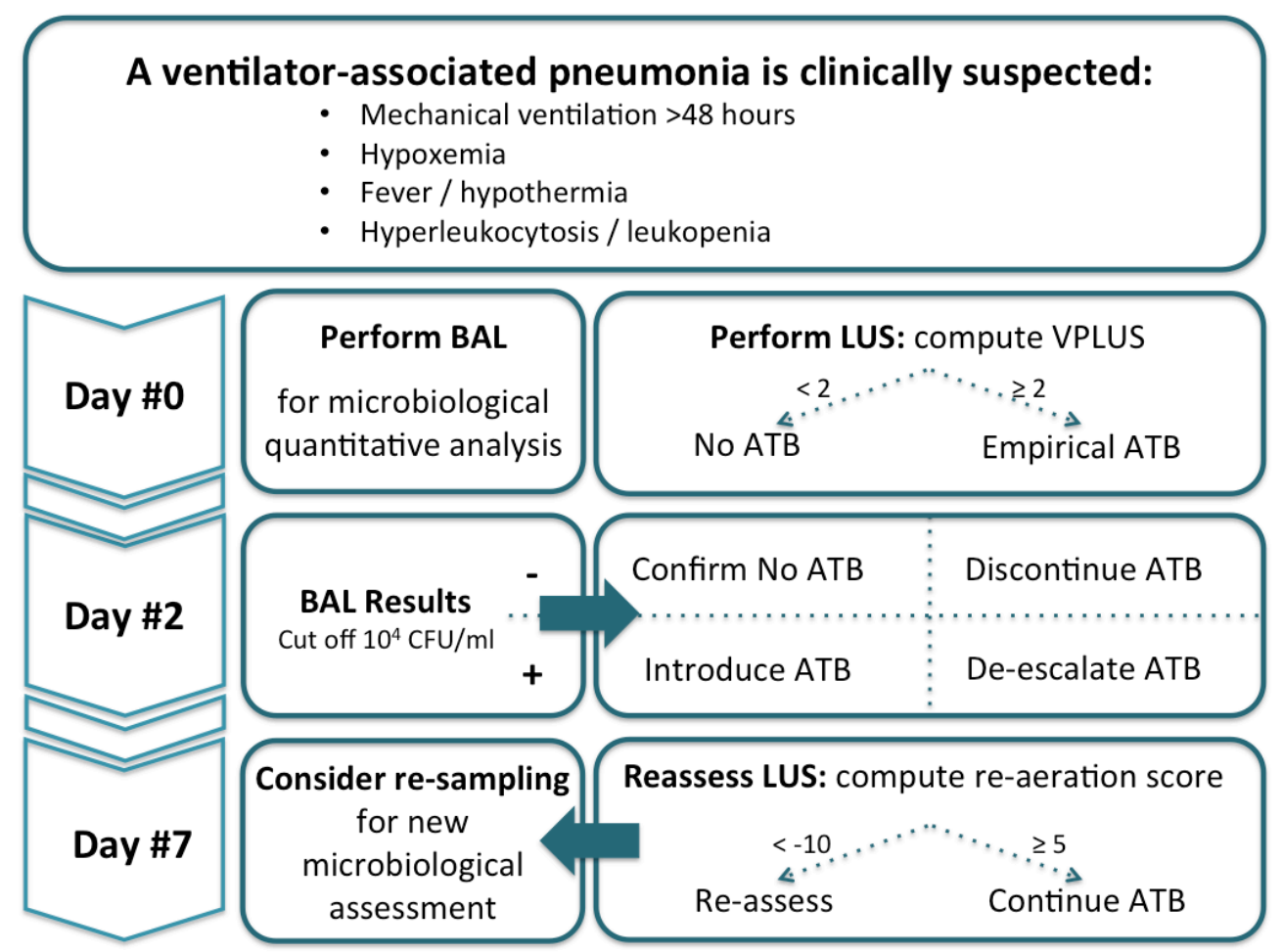

Figure 6 A systematic approach for diagnosis and monitoring of ventilator-associated pneumonia. BAL, broncho-alveolar lavage; ATB, antibiotics; VPLUS, ventilator-associated pneumonia lung ultrasound score; LUS, lung ultrasound.
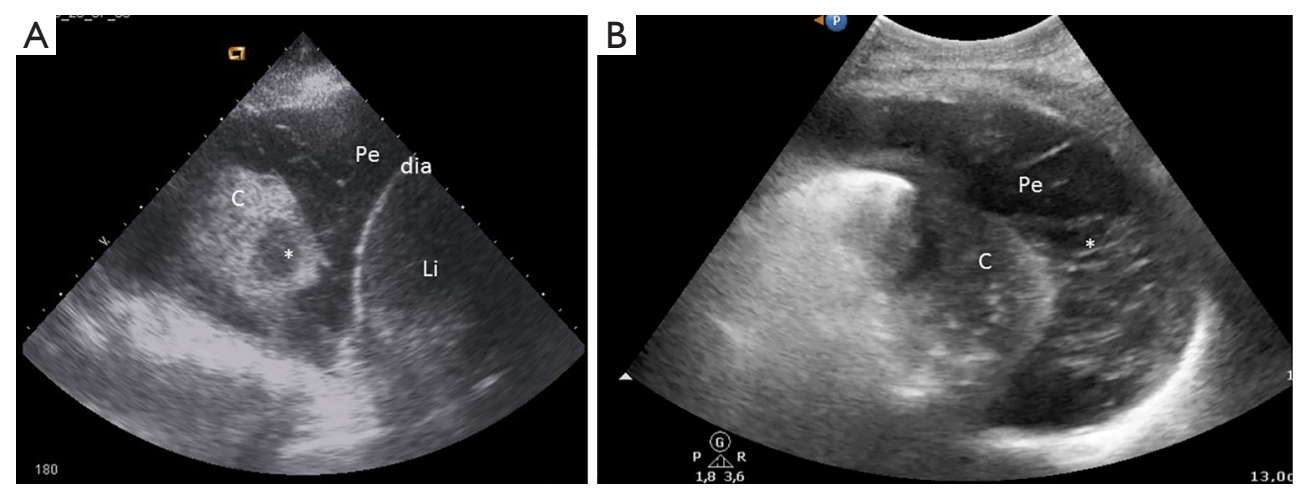

Figure 7 Lung abscesses and adhesiolysis. (A) Frontal view of lung abscesses $\left(^{*}\right)$ inside a lung consolidation (C); (B) transversal view of a lung consolidation with pleural effusion $(\mathrm{Pe})$ and adhesiolysis $\left(^{*}\right)$. Pe, pleural effusion; dia, diaphragm; Li, liver.

\section{Acknowledgements}

Support was provided solely from departmental sources.

\section{Footnote}

Conflicts of Interest: The authors have no conflicts of interest to declare.

\section{References}

1. Kollef MH, Hamilton CW, Ernst FR. Economic impact of ventilator-associated pneumonia in a large matched cohort. Infect Control Hosp Epidemiol 2012;33:250-6.

2. Melsen WG, Rovers MM, Groenwold RH, et al.

Attributable mortality of ventilator-associated pneumonia: a meta-analysis of individual patient data from randomised 
prevention studies. Lancet Infect Dis 2013;13:665-71.

3. Lipovy B, Rihova H, Gregorova N, et al. Epidemiology of ventilator-associated tracheobronchitis and vantilatorassociated pneumonia in patients with inhalation injury at the Burn Centre in Brno (Czech Republic). Ann Burns Fire Disasters 2011;24:120-5.

4. Rosenthal VD, Bijie H, Maki DG, et al. International Nosocomial Infection Control Consortium (INICC) report, data summary of 36 countries, for 2004-2009. Am J Infect Control 2012;40:396-407.

5. Tejerina E, Esteban A, Fernandez-Segoviano P, et al. Accuracy of clinical definitions of ventilator-associated pneumonia: comparison with autopsy findings. J Crit Care 2010;25:62-8.

6. Chastre J, Luyt CE. Does this patient have VAP? Intensive Care Med 2016;42:1159-63.

7. Pugin J, Auckenthaler R, Mili N, et al. Diagnosis of ventilator-associated pneumonia by bacteriologic analysis of bronchoscopic and nonbronchoscopic "blind" bronchoalveolar lavage fluid. Am Rev Respir Dis 1991;143:1121-9.

8. Luna CM, Aruj P, Niederman MS, et al. Appropriateness and delay to initiate therapy in ventilator-associated pneumonia. Eur Respir J 2006;27:158-64.

9. Rello J, Paiva JA, Baraibar J, et al. International Conference for the Development of Consensus on the Diagnosis and Treatment of Ventilator-associated Pneumonia. Chest 2001;120:955-70.

10. Lefcoe MS, Fox GA, Leasa DJ, et al. Accuracy of portable chest radiography in the critical care setting. Diagnosis of pneumonia based on quantitative cultures obtained from protected brush catheter. Chest 1994;105:885-7.

11. Wunderink RG, Woldenberg LS, Zeiss J, et al. The radiologic diagnosis of autopsy-proven ventilatorassociated pneumonia. Chest 1992;101:458-63.

12. American Thoracic S, Infectious Diseases Society of A. Guidelines for the management of adults with hospitalacquired, ventilator-associated, and healthcare-associated pneumonia. Am J Respir Crit Care Med 2005;171:388-416.

13. Eccles S, Pincus C, Higgins B, et al. Diagnosis and management of community and hospital acquired pneumonia in adults: summary of NICE guidance. BMJ 2014;349:g6722.

14. Bouhemad B, Mongodi S, Via G, et al. Ultrasound for "lung monitoring" of ventilated patients. Anesthesiology 2015;122:437-47.

15. Mayo PH, Beaulieu Y, Doelken P, et al. American College of Chest Physicians/La Societe de Reanimation de Langue
Francaise statement on competence in critical care ultrasonography. Chest 2009;135:1050-60.

16. Zieleskiewicz L, Muller L, Lakhal K, et al. Point-of-care ultrasound in intensive care units: assessment of 1073 procedures in a multicentric, prospective, observational study. Intensive Care Med 2015;41:1638-47.

17. Laursen CB, Sloth E, Lassen AT, et al. Point-of-care ultrasonography in patients admitted with respiratory symptoms: a single-blind, randomised controlled trial. Lancet Respir Med 2014;2:638-46.

18. Fabregas N, Ewig S, Torres A, et al. Clinical diagnosis of ventilator associated pneumonia revisited: comparative validation using immediate post-mortem lung biopsies. Thorax 1999;54:867-73.

19. Fartoukh M, Maitre B, Honore S, et al. Diagnosing pneumonia during mechanical ventilation: the clinical pulmonary infection score revisited. Am J Respir Crit Care Med 2003;168:173-9.

20. Jung B, Embriaco N, Roux F, et al. Microbiogical data, but not procalcitonin improve the accuracy of the clinical pulmonary infection score. Intensive Care Med 2010;36:790-8.

21. Kalil AC, Metersky ML, Klompas M, et al. Management of Adults With Hospital-acquired and Ventilator-associated Pneumonia: 2016 Clinical Practice Guidelines by the Infectious Diseases Society of America and the American Thoracic Society. Clin Infect Dis 2016;63:e61-e111.

22. Rotstein C, Evans G, Born A, et al. Clinical practice guidelines for hospital-acquired pneumonia and ventilatorassociated pneumonia in adults. Can J Infect Dis Med Microbiol 2008;19:19-53.

23. Nseir S, Favory R, Jozefowicz E, et al. Antimicrobial treatment for ventilator-associated tracheobronchitis: a randomized, controlled, multicenter study. Crit Care 2008;12:R62.

24. Winer-Muram HT, Steiner RM, Gurney JW, et al. Ventilator-associated pneumonia in patients with adult respiratory distress syndrome: CT evaluation. Radiology 1998;208:193-9.

25. Chiumello D, Mongodi S, Algieri I, et al. Assessment of Lung Aeration and Recruitment by CT Scan and Ultrasound in Acute Respiratory Distress Syndrome Patients. Crit Care Med 2018.

26. Soldati G, Inchingolo R, Smargiassi A, et al. Ex vivo lung sonography: morphologic-ultrasound relationship. Ultrasound Med Biol 2012;38:1169-79.

27. Volpicelli G, Elbarbary M, Blaivas M, et al. International evidence-based recommendations for point-of-care lung 
ultrasound. Intensive Care Med 2012;38:577-91.

28. Lichtenstein D, Meziere G, Seitz J. The dynamic air bronchogram. A lung ultrasound sign of alveolar consolidation ruling out atelectasis. Chest 2009;135:1421-5.

29. Bouhemad B, Dransart-Rayé O, Mojoli F, et al. Longitudinal scan with linear probe. Asvide 2018;5:853. Available online: http://www.asvide.com/article/ view/28249

30. Bouhemad B, Dransart-Rayé O, Mojoli F, et al. Longitudinal scan with phased-array probe. Asvide 2018;5:854. Available online: http://www.asvide.com/ article/view/28250

31. Claessens YE, Debray MP, Tubach F, et al. Early Chest Computed Tomography Scan to Assist Diagnosis and Guide Treatment Decision for Suspected Communityacquired Pneumonia. Am J Respir Crit Care Med 2015;192:974-82.

32. Lichtenstein DA, Meziere GA. Relevance of lung ultrasound in the diagnosis of acute respiratory failure: the BLUE protocol. Chest 2008;134:117-25.

33. Bourcier JE, Paquet J, Seinger M, et al. Performance comparison of lung ultrasound and chest $\mathrm{x}$-ray for the diagnosis of pneumonia in the ED. Am J Emerg Med 2014;32:115-8.

34. Cortellaro F, Colombo S, Coen D, et al. Lung ultrasound is an accurate diagnostic tool for the diagnosis of pneumonia in the emergency department. Emerg Med J 2012;29:19-23.

35. Liu XL, Lian R, Tao YK, et al. Lung ultrasonography: an effective way to diagnose community-acquired pneumonia. Emerg Med J 2015;32:433-8.

36. Pagano A, Numis FG, Visone G, et al. Lung ultrasound for diagnosis of pneumonia in emergency department. Intern Emerg Med 2015;10:851-4.

37. Parlamento S, Copetti R, Di Bartolomeo S. Evaluation of lung ultrasound for the diagnosis of pneumonia in the ED. Am J Emerg Med 2009;27:379-84.

38. Reissig A, Copetti R, Mathis G, et al. Lung ultrasound in the diagnosis and follow-up of community-acquired pneumonia: a prospective, multicenter, diagnostic accuracy study. Chest 2012;142:965-72.

39. Reissig A, Kroegel C. Sonographic diagnosis and followup of pneumonia: a prospective study. Respiration 2007;74:537-47.

40. Sperandeo M, Carnevale V, Muscarella S, et al. Clinical application of transthoracic ultrasonography in inpatients with pneumonia. Eur J Clin Invest 2011;41:1-7.

41. Long L, Zhao HT, Zhang ZY, et al. Lung ultrasound for the diagnosis of pneumonia in adults: A meta-analysis. Medicine (Baltimore) 2017;96:e5713.

42. Llamas-Alvarez AM, Tenza-Lozano EM, Latour-Perez J. Accuracy of Lung Ultrasonography in the Diagnosis of Pneumonia in Adults: Systematic Review and MetaAnalysis. Chest 2017;151:374-82.

43. Gallard E, Redonnet JP, Bourcier JE, et al. Diagnostic performance of cardiopulmonary ultrasound performed by the emergency physician in the management of acute dyspnea. Am J Emerg Med 2015;33:352-8.

44. Liteplo AS, Marill KA, Villen T, et al. Emergency thoracic ultrasound in the differentiation of the etiology of shortness of breath (ETUDES): sonographic B-lines and N-terminal pro-brain-type natriuretic peptide in diagnosing congestive heart failure. Acad Emerg Med 2009;16:201-10.

45. Zagli G, Cozzolino M, Terreni A, et al. Diagnosis of ventilator-associated pneumonia: a pilot, exploratory analysis of a new score based on procalcitonin and chest echography. Chest 2014;146:1578-85.

46. Berlet T, Etter R, Fehr T, et al. Sonographic patterns of lung consolidation in mechanically ventilated patients with and without ventilator-associated pneumonia: a prospective cohort study. J Crit Care 2015;30:327-33.

47. Mongodi S, Via G, Girard M, et al. Lung Ultrasound for Early Diagnosis of Ventilator-Associated Pneumonia. Chest 2016;149:969-80.

48. Bouhemad B, Liu ZH, Arbelot C, et al. Ultrasound assessment of antibiotic-induced pulmonary reaeration in ventilator-associated pneumonia. Crit Care Med 2010;38:84-92.

49. Mongodi S, Bouhemad B, Orlando A, et al. Modified Lung Ultrasound Score for Assessing and Monitoring Pulmonary Aeration. Ultraschall Med 2017;38:530-7.

50. Soummer A, Perbet S, Brisson H, et al. Ultrasound assessment of lung aeration loss during a successful weaning trial predicts postextubation distress* ${ }^{*}$ Crit Care Med 2012;40:2064-72.

51. Mongodi S, Pozzi M, Orlando A, et al. Lung ultrasound for daily monitoring of ARDS patients on extracorporeal membrane oxygenation: preliminary experience. Intensive Care Med 2018;44:123-4.

52. Caltabeloti F, Monsel A, Arbelot C, et al. Early fluid loading in acute respiratory distress syndrome with septic shock deteriorates lung aeration without impairing arterial oxygenation: a lung ultrasound observational study. Crit Care 2014;18:R91.

53. Bouhemad B, Brisson H, Le-Guen M, et al. Bedside 
ultrasound assessment of positive end-expiratory pressureinduced lung recruitment. Am J Respir Crit Care Med 2011;183:341-7.

54. Rouby JJ, Arbelot C, Gao Y, et al. Training for Lung Ultrasound Score Measurement in Critically Ill Patients. Am J Respir Crit Care Med 2018.

55. Gehmacher O, Mathis G, Kopf A, et al. Ultrasound imaging of pneumonia. Ultrasound Med Biol 1995;21:1119-22.

56. Klein JS, Schultz S, Heffner JE. Interventional radiology of the chest: image-guided percutaneous drainage of pleural effusions, lung abscess, and pneumothorax. AJR
Am J Roentgenol 1995;164:581-8.

57. Yang PC, Chang DB, Yu CJ, et al. Ultrasound guided percutaneous cutting biopsy for the diagnosis of pulmonary consolidations of unknown aetiology. Thorax 1992;47:457-60.

58. Yang PC, Luh KT, Lee YC, et al. Lung abscesses: US examination and US-guided transthoracic aspiration. Radiology 1991;180:171-5.

59. Mongodi S, Bouhemad B, Orlando A, et al. Lung ultrasound before and after fiberbronchoscopy -modifications may improve ventilator-associated pneumonia diagnosis. Crit Care 2016;20:P094.
Cite this article as: Bouhemad B, Dransart-Rayé O, Mojoli F, Mongodi S. Lung ultrasound for diagnosis and monitoring of ventilator-associated pneumonia. Ann Transl Med 2018;6(21):418. doi: $10.21037 /$ atm.2018.10.46 